\title{
REMARKS ON CYCLIC ADDITIVITY
}

\author{
J. W. T. YOUNGS ${ }^{1}$
}

1. Introduction. For the purposes of this discussion suppose that $X$ and $Y$ are topological spaces while $G$ is a commutative, topological semi-group (with zero element) which, as a space, is Hausdorff. In other words, each pair $\left(g_{1}, g_{2}\right)$ of elements in $G$ uniquely determines an element $\left(g_{1}+g_{2}\right)$ in $G$; the operation + is associative and commutative; there is a unique element 0 such that $g \in G$ implies $g+0=g$; finally, $G$ is a Hausdorf space and the operation + provides a mapping (=continuous transformation) from the product space $G \times G$ into $G$. Obviously, topological groups, and the space of non-negative real numbers compactified by the addition of $\infty$, with the operation + meaning addition, and the convention that $a+\infty=\infty+a=\infty$, provide examples of such semi-groups.

It will be said that $l m$ is a Peanian factorization of a mapping $f: X \rightarrow Y$ if and only if there are mappings $m: X \rightarrow \mathfrak{X}$ and $l: \mathfrak{X} \rightarrow Y$ such that $\mathfrak{X}$ is a Peano space and the composition $l m$ is $f$. The space $\mathfrak{X}$ is called the middle space of the Peanian factorization $l m$ of $f$.

Let $F$ be the class of mappings $f: X \rightarrow Y$ each of which has at least one Peanian factorization, and suppose that $\gamma$ is a transformation from $F$ into $G$.

For each Peano space $\mathfrak{X}$ let $\mathcal{E}(\mathfrak{X})$ be the class of true cyclic elements of $\mathfrak{X}$. (See Whyburn [6] for the Peano space theory involved in this paper.) $)^{2}$ If $\mathbb{E} \in \mathcal{E}(\mathfrak{X})$ there is a unique monotone retraction $r \mathbb{E}: \mathfrak{X} \rightrightarrows(\mathbb{E}$. (The double arrow indicates that $r_{\mathbb{E}}(\mathfrak{X})=\mathbb{E}$.) If $f \in F$ and $l m$ is a Peanian factorization of $f$ with middle space $\mathfrak{X}$, while $\mathfrak{E} \in \mathcal{E}(\mathfrak{X})$, then define $f_{\circledast}=l r \Subset m$.

It is the object of this paper to investigate the statement

$$
\gamma(f)=\sum \gamma\left(f_{\mathfrak{E}}\right), \quad \quad \mathfrak{E} \in \mathcal{E}(\mathfrak{X})
$$

where the equality means that for each neighborhood $U$ of $\gamma(f)$ there is a finite subclass $\mathcal{F}(U)$ of $\mathcal{E}(\mathfrak{X})$ such that if $\mathcal{F}$ is any finite subclass of $\mathcal{E}(\mathfrak{X})$ containing $\mathcal{F}(U)$ then $U \ni \sum \gamma\left(f_{\mathfrak{E}}\right)$, $\mathbb{E} \in \mathcal{F}$, it being understood that addition over an empty class yields 0 .

In the event that (1) holds for each $f \in F$ and for each Peanian factorization of $f$, then $\gamma$ is said to be cyclicly additive.

Cyclic additivity theorems of a weaker type have been considered

Received by the editors April 11, 1948.

${ }^{1}$ Fellow of the John Simon Guggenheim Memorial Foundation.

2 Numbers in brackets refer to the bibliography at the end of the paper. 
by Helsel [3], Radó [5] and others in connection with Lebesgue area.

2. The theorems. The first object is to investigate consequences of the assumption that $\gamma$ is cyclicly additive.

I. If $f \in F$ and has a Peanian factorization in which the middle space is a dendrite, then $\gamma(f)=0$.

This is an obvious consequence of the fact that a dendrite has no true cyclic elements.

$\mathrm{I}_{\mathrm{a}}$. If $f \in F$ and has a Peanian factorization in which the middle space is an arc, then $\gamma(f)=0$.

(It is understood that an arc may consist of a single point.)

Before considering more substantial necessary conditions, suppose that $\mathfrak{X}$ is a Peano space and define $\mathfrak{B} \in P(\mathfrak{X})$ if and only if $\mathfrak{B}$ is a Peano subspace of $\mathfrak{X}$ such that $\mathcal{E}(\mathfrak{P}) \subset \mathcal{E}(\mathfrak{X})$. Let $\mathfrak{Q} \in \mathscr{Q}(\mathfrak{X})$ if and only if $\mathfrak{Q}$ is a Peano subspace of $\mathfrak{X}$ having a finite cyclic chain approximation $\mathfrak{E}_{1}, \cdots, \mathfrak{E}_{q}$ such that either $\mathfrak{E}_{k}$ is an arc, or $\mathfrak{\mho}_{k} \in \mathcal{E}(\mathfrak{X}), k=1, \cdots, q$. (See Whyburn [6, p. 73].)

It is easy to see that $2(\mathfrak{X}) \subset \mathscr{P}(\mathfrak{X}) \supset \mathcal{A}(\mathfrak{X})$, where $\mathcal{A}(\mathfrak{X})$ is the class of $A$-sets in $\mathfrak{X}$.

Given $\mathfrak{P} \in \mathbb{P}(\mathfrak{X})$, let $\mathfrak{A}=\mathfrak{P} \cup \cup \mathfrak{E}$, where the union is taken over those elements $\mathbb{E} \in \mathcal{E}(\mathfrak{X})$ having the property that $\mathfrak{B} \cap \mathbb{E}$ consists of at least two points. It follows that $\mathfrak{A} \in \mathcal{A}(\mathfrak{X})$, that $x \in \mathfrak{A}-\mathfrak{P}$ implies there is a unique $\mathbb{E} \in \mathcal{E}(\mathfrak{U})$ such that $\mathfrak{x} \in \mathbb{E}$, and if $\mathcal{E}(\mathfrak{P}) \boxplus \mathbb{E} \in \mathcal{E}(\mathfrak{H})$ then $\mathscr{E} \cap \Re$ is a dendrite. Since a dendrite is an absolute retract if $\mathcal{E}(\mathfrak{P}) \boxplus \mathbb{E} \in \mathcal{E}(\mathfrak{A})$, then there is a retraction $\rho \mathfrak{E}: \mathbb{E} \rightarrow(\mathbb{E} \cap \mathfrak{P})$. (See Borsuk [1].) Define

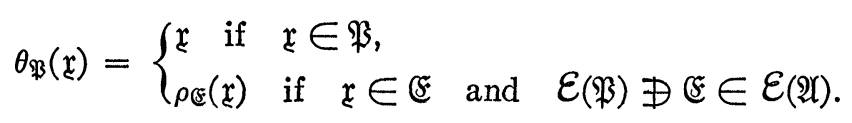

It follows that $\theta_{\mathfrak{B}}$ is a retraction from $\mathfrak{A}$ onto $\mathfrak{B}$, but $\theta_{\mathfrak{B}}$ is not uniquely defined in terms of $\mathfrak{A}$ and $\mathfrak{B}$. If $r_{\mathfrak{A}}: \mathfrak{X} \rightarrow \mathfrak{A}$ is the unique monotone retraction, and $r_{\mathfrak{B}}=\theta_{\mathfrak{F}} r_{\mathfrak{A}}$, then $r_{\mathfrak{P}}: \mathfrak{X} \rightrightarrows \mathfrak{B}$ is a retraction.

The retraction $r_{\mathfrak{P}}$ depends upon the retraction $\theta_{\mathfrak{B}}$ which is not

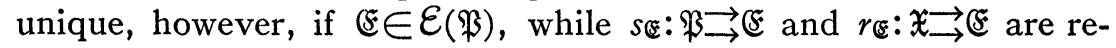
tractions, then

$$
r_{\mathbb{E}}=s_{\mathscr{E}} r_{\mathfrak{B}} .
$$

There may be retractions from $\mathfrak{X}$ onto $\mathfrak{P}$ defined in other ways; however, the notation $r_{\mathfrak{B}}$ will always be used to indicate a retraction $\theta_{\mathfrak{B}} r_{\mathfrak{X}}$ defined as above, hence $r_{\mathfrak{B}}$ is unique modulo the factor $\theta_{\mathfrak{P}}$.

In this connection notice that if $\alpha$ is the maximum of the diameters 
of the components of $\mathfrak{X}-\mathfrak{A}$, and $\beta$ is the maximum of the diameters of $\mathbb{E}$ for $\mathcal{E}(\mathfrak{P}) \boxplus \in \mathcal{E}(\mathfrak{U})$, then

$$
\rho\left\{\mathfrak{x}, r_{\mathfrak{B}}(\mathfrak{x})\right\} \leqq \alpha+\beta .
$$

If $f \in F$ and $l m$ is a Peanian factorization of $f$ with middle space $\mathfrak{X}$ while $\mathfrak{B} \in \mathcal{P}(\mathfrak{X})$, then $f_{\mathfrak{P}}=l_{r_{\mathfrak{B}} m}$ is a mapping from $X$ into $Y$ having a Peanian factorization $l\left(r_{\mathfrak{B}} m\right)$ with middle space $\mathfrak{P}$. In other words, $f_{\Re} \in F$. Different selections of $r_{\mathfrak{B}}$ will produce different mappings $f_{\mathfrak{B}}$; however, if $\mathbb{E} \in \mathcal{E}(\mathfrak{P})$ then in view of (2) it is true that $f_{\mathbb{E}} \equiv \operatorname{lr} \mathbb{E}$ $=l_{S \Subset} r_{\mathfrak{P}} m \equiv\left(f_{\mathfrak{B}}\right)_{\mathfrak{E}} \equiv f_{\mathfrak{B}}$. Hence $f_{\mathfrak{B}}$ is independent of the retraction $r_{\mathfrak{P}}$ in spite of the fact that $f_{\mathfrak{P}}$ is not.

II. If $f \in F$ has a Peanian factorization $l m$ with middle space $\mathfrak{X}$, while $\mathfrak{A}_{i} \in \mathcal{C}(\mathfrak{X}), i=1,2, \mathfrak{A}_{1} \cap \mathfrak{A}_{2}$ is a point, and $\mathfrak{V}_{1} \cup \mathfrak{A}_{2}=\mathfrak{X}$, then $\gamma\left(f_{\mathfrak{I}_{1}}\right)+\gamma\left(f_{\mathfrak{P}_{2}}\right)=\gamma(f)$.

PROoF. If the theorem is false suppose that the sum on the left above is $\eta \neq \gamma(f)$, and select neighborhoods $U$ and $U_{0}$ for $\eta$ and $\gamma(f)$, respectively, such that

$$
U \cap U_{0}=\varnothing \text {. }
$$

Let $U_{i}$ be a neighborhood of $\gamma\left(f_{\mathfrak{T}_{i}}\right), i=1,2$, such that

$$
U_{1}+U_{2} \subset U \text {. }
$$

Note that $\mathcal{E}\left(\mathfrak{A}_{1}\right) \cup \mathcal{E}\left(\mathfrak{A}_{2}\right)=\mathcal{E}(\mathfrak{X})$, and $\mathcal{E}\left(\mathfrak{A}_{1}\right) \cap \mathcal{E}\left(\mathfrak{A}_{2}\right)=\varnothing$. Since $\mathcal{A}(\mathfrak{X})$ $\subset \mathscr{P}(\mathfrak{X})$ and $\gamma$ is cyclicly additive, there is a finite subcollection $\mathcal{F}\left(U_{i}\right)$ of $\mathcal{E}\left(\mathfrak{A}_{i}\right)$ such that if $\mathcal{F}_{i}$ is a finite subcollection of $\mathcal{E}\left(\mathfrak{A}_{i}\right)$ containing $\mathcal{F}\left(U_{i}\right)$, then (understanding that $\mathfrak{X}_{0}=\mathfrak{X}$ )

$$
U_{i} \ni \sum \gamma\left(f_{\circledast}\right), \quad \Subset \in \mathcal{F}_{i}, \quad i=0,1,2 .
$$

If $\mathcal{F}$ is any finite subcollection of $\mathcal{E}(\mathfrak{X})$ containing $\mathcal{F}\left(U_{0}\right) \cup \mathcal{F}\left(U_{1}\right)$ $\cup \mathcal{F}\left(U_{2}\right)$ let $\mathcal{F}_{i}=\mathcal{F} \cap \mathcal{E}\left(\mathfrak{A}_{i}\right), i=1,2$, and note that $\mathcal{F}_{1} \cap \mathcal{F}_{2}=\varnothing$ while $\mathcal{F}_{1} \cup \mathcal{F}_{2}=\mathcal{F}$. Hence

$$
\begin{aligned}
U_{0} & \ni\left[\sum \gamma\left(f_{\mathfrak{E}}\right), \notin \in \mathcal{F}\right] \\
& =\left[\sum \gamma\left(f_{\mathfrak{E}}\right), \notin \in \mathcal{F}_{1}\right]+\left[\sum \gamma\left(f_{\mathfrak{E}}\right), \mathfrak{E} \in \mathcal{F}_{2}\right] \\
& \in U_{1}+U_{2} \subset U .
\end{aligned}
$$

Therefore $U \cap U_{0} \neq \varnothing$ in contradiction to (4).

$\mathrm{II}_{\mathrm{a}}$. The same as II except that the hypothesis is strengthened by the condition $\mathfrak{X} \in \mathscr{Q}(\mathfrak{X})$.

III. If $f \in F$ and has a Peanian factorization $l m$ with middle space $\mathfrak{X}$, then for each neighborhood $U$ of $\gamma(f)$ there is a $\mathfrak{Q} \in \mathcal{Q}(\mathfrak{X})$ such that $\mathfrak{Q} \subset \mathfrak{P} \in \mathbb{P}(\mathfrak{X})$ implies that $\gamma\left(f_{\mathfrak{B}}\right) \in \mathfrak{U}$. 
Proof. There is a finite subcollection $\mathcal{f}(U)$ of $\mathcal{E}(\mathfrak{X})$ such that if $\mathcal{f}$ is any finite subcollection of $\mathcal{E}(\mathfrak{X})$ containing $\mathcal{F}(U)$, then $U \ni \sum \gamma\left(f_{\mathscr{E}}\right)$, $\& \in \mathcal{F}$. The theorem will be proved if it can be shown that there is a $\mathfrak{Q} \in \mathcal{Q}(\mathfrak{X})$ such that $\mathcal{E}(\mathfrak{Q})=\mathfrak{F}(U)$.

If $\mathcal{F}(U)=\varnothing$ let $\mathfrak{Q}$ be any point in $\mathfrak{X}$. If $\mathcal{F}(U) \neq \varnothing$, suppose $\mathbb{E}_{1}, \cdots, \mathbb{E}_{n}$ are the elements in $\mathcal{F}(U)$. Define $\mathfrak{E}_{1}{ }^{*}=\mathbb{E}_{1}$ and join $\mathbb{E}_{2}$ to $\mathfrak{G}_{1}{ }^{*}$ by an arc $\Omega_{1}$ with end points only in $\mathbb{E}_{1}^{*} \cup \mathbb{E}_{2}$. The arc $\Omega_{1}$ may be a single point. Let $\mathfrak{E}_{2}^{*}=\mathfrak{E}_{2} \cup \Omega_{1} \cup \cup \mathbb{E}$ where the union is taken over the true cyclic elements $\&$ in $\mathcal{F}(U)$ which have at least two points in common with $\Omega_{1}$.

There is a first subscript $n_{1}$, if any, for which $\mathbb{E}_{n_{1}}$ is not in $\mathfrak{E}_{1}{ }^{*} \cup \mathfrak{E}_{2}{ }^{*}$. Join $\mathfrak{E}_{n_{1}}$ to $\mathfrak{E}_{1}^{*} \cup \mathfrak{E}_{2}^{*}$ by an arc $\Omega_{2}$ with end points only in $\mathfrak{E}_{1}^{*} \cup \mathfrak{E}_{2}^{*} \cup \mathfrak{E}_{n_{1}}$. Let $\mathfrak{E}_{3}^{*}=\mathfrak{E}_{n_{1}} \cup \Re_{2} \cup \cup \notin$ where the union is taken over the true cyclic elements $\mathbb{E}$ in $\mathcal{F}(U)$ which have at least two points in common with $\Omega_{2}$.

This process stops after a finite number of steps $m$ for want of a true cyclic element in $\mathcal{F}(U)$ not in $\mathfrak{E}_{1}{ }^{*} \cup \ldots \cup \mathbb{C}_{m}^{*} \equiv \mathfrak{Q}$. The Peano space $\mathfrak{Q}$ certainly has a cyclic chain approximation $\mathfrak{E}_{1}, \cdots, \mathfrak{E}_{q}$ such that $\mathfrak{E}_{k}$ is either an arc or $\mathfrak{S}_{k} \in \mathcal{F}(U) \subset \mathcal{E}(\mathfrak{X}), k=1, \cdots, q$. Hence $\mathfrak{Q} \in \mathcal{Q}(\mathfrak{X})$ and $\mathcal{E}(\mathfrak{Q})=\mathscr{F}(U)$.

III $_{\mathrm{a}}$. If $f \in F$ and has a Peanian factorization $l m$ with middle space $\mathfrak{X}$, then for each neighborhood $U$ of $\gamma(f)$ there is a $\mathfrak{Q} \in \mathscr{Q}(\mathfrak{X})$ such that $\mathfrak{Q} \subset \Re \in \mathscr{Q}(\mathfrak{X})$ implies $\gamma\left(f_{\Re}\right) \in U$.

The question of the sufficiency of these conditions is considered next.

THEOREM. If $\gamma$ is a transformation from $F$ into $G$ having the properties $\mathrm{I}_{\mathrm{a}}, \mathrm{II}_{\mathrm{a}}$ and $\mathrm{III}_{\mathrm{a}}$, then $\gamma$ is cyclicly additive.

Proof. Let $f \in F$ and suppose $l m$ is a Peanian factorization of $f$ with middle space $\mathfrak{X}$. Suppose $U$ is a neighborhood of $\gamma(f)$ and let $\mathfrak{Q}$ be the space in $2(\mathfrak{X})$ given by $\operatorname{III}_{\mathfrak{a}}$. Define $\mathcal{f}(U)=\mathcal{E}(\mathfrak{Q})$, and suppose $\mathcal{F}$ is a finite subcollection of $\mathcal{E}(\mathfrak{X})$ containing $\mathcal{F}(U)$.

If $\mathcal{F}=\mathcal{F}(U)$ let $\mathfrak{R}=\mathfrak{Q}$. If $\mathcal{F} \neq \mathcal{F}(U)$, the construction used in the proof of III shows that there is an $\Re \in \mathcal{Q}(\mathfrak{X})$ such that $\mathcal{E}(\Re)=\mathcal{F}$ and $\Re \supset \mathfrak{Q}$. By $\operatorname{III}_{\mathfrak{a}}, \gamma\left(f_{\Re}\right) \in U$.

But $\Re$ has a finite cyclic chain decomposition $\mathfrak{G}_{1}, \cdots, \mathfrak{C}_{r}$ such that either $\mathfrak{E}_{k}$ is an arc or $\mathfrak{E}_{k} \in \mathcal{E}(\mathfrak{X}), k=1, \cdots, r$. Using the properties of a cyclic chain approximation, together with $I_{a}$ and $I_{a}$ it follows directly that

$$
\begin{aligned}
& \gamma\left(f_{\Re}\right)=\sum \gamma\left(f_{\Re(E}\right), \quad \quad \in \in \mathcal{E}(\Re), \\
& =\sum \gamma\left(f_{\circledast}\right), \quad \notin \in \mathcal{F} \text {. }
\end{aligned}
$$


Therefore,

$$
U \ni \sum \gamma\left(f_{\mathfrak{E}}\right)
$$

and hence

$$
\gamma(f)=\sum \gamma\left(f_{\mathfrak{E}}\right), \quad \quad \& \in \mathcal{E}(\mathfrak{X}) .
$$

3. An application. Though greater generality is possible, suppose that $X$ and $Y$ are compacta, and for fixed $q \geqq 0$ consider the Cech cohomology groups $H^{q}(X)$ and $H^{q}(Y)$ where the coefficient group is discrete and does not change in the remainder of the discussion. The topology on $H^{q}(X)$ and $H^{q}(Y)$ is taken discrete and it should be mentioned that if $q=0$ one considers the reduced cohomology group.

If $f \in F$ then there is an induced homomorphism

$$
f^{*}: H^{q}(Y) \rightarrow H^{q}(X) .
$$

Select any element $y \in H^{q}(Y)$, let $G=H^{q}(X)$, and define

$$
\gamma(f)=f^{*}(y) \text {. }
$$

It will be shown that $\gamma$ is cyclicly additive; that is, if $\operatorname{lm}$ is a Peanian factorization of $f \in F$ with middle space $\mathfrak{X}$, then for each $y \in H^{q}(Y)$,

$$
f^{*}(y)=\sum f_{\mathfrak{E}}^{*}(y), \quad \xi \in \mathcal{E}(\mathfrak{X}) .
$$

In view of the sufficiency theorem it is enough to check conditions $\mathrm{I}_{\mathrm{a}}, \mathrm{II}_{\mathrm{a}}$ and $\mathrm{III}_{\mathrm{a}}$.

In the event $f \in F$ and has a Peanian factorization $l m$ whose middle space is an arc, then $f$ is clearly homotopic to a point and hence $f^{*}(y)=0$.

To check $\mathrm{II}_{\mathrm{a}}$ one will need the fact that: If $X_{1}$ and $X_{2}$ are closed subsets of $X$, and $X=X_{1} \cup X_{2}$, while $f_{i}: X \rightarrow Y$ is a mapping for $i=0,1,2$, such that $f_{0}=f_{i}$ on $X_{i}, i=1,2$, and $x \in X_{j}$ implies $f_{i}(x)=y_{0}$, a single point of $Y, i, j=1,2 ; i \neq j$; then $f_{0}^{*}=f_{1}^{*}+f_{2}^{*}$. (See Borsuk [2].)

Suppose $\mathfrak{A}_{i} \in \mathcal{C}(\mathfrak{X}), i=1,2$, and $\mathfrak{A}_{1} \cap \mathfrak{P}_{2}$ is a point $\mathfrak{x}_{0}$, while $\mathfrak{A}_{1} \cup \mathfrak{A}_{2}$ $=\mathfrak{X}$. Define $X_{i}=m^{-1}\left(\mathfrak{A}_{i}\right)$ and $f_{i}=l_{r \mathfrak{r}_{i}} m, i=1,2$. Now $X_{1}$ and $X_{2}$ are closed subsets of $X$ while $X_{1} \cup X_{2}=X$. Moreover, if $x \in X_{i}$, then $m(x)$ $\in \mathfrak{A}_{i}$, hence $r_{\mathfrak{A}_{i}} m(x)=m(x)$ and so $f_{i}(x)=l m(x)=f(x)$; however, $f_{j}(x)$ $=l r_{\mathfrak{R}_{j}} m(x)=l\left(\mathfrak{x}_{0}\right)$ since $r_{\mathfrak{A}_{j}}\left(\mathfrak{A}_{i}\right)=\mathfrak{x}_{0}, i, j=1,2 ; i \neq j$. Hence $f^{*}=f_{1}^{*}+f_{2}{ }^{*}$ and $\gamma(f)=\gamma\left(f_{\mathfrak{A}_{1}}\right)+\gamma\left(f_{\mathfrak{A}_{2}}\right)$, which shows that condition II and hence $\mathrm{II}_{\mathrm{a}}$ is satisfied.

To check condition $\operatorname{III}_{\mathrm{a}}$ recall that there is an $\epsilon(y)$ such that if $\phi_{1}: X \rightarrow Y$ and $\phi_{2}: X \rightarrow Y$ are two mappings with the property that $\rho\left\{\phi_{1}(x), \phi_{2}(x)\right\}<\epsilon(y), x \in X$, then $\phi_{1}^{*}(y)=\phi_{2}^{*}(y)$. (See, for example, Hurewicz-Wallman [4, p. 140].) 
Since $l$ is continuous on a compact space there is a $\delta$ such that $\rho\left\{\mathfrak{x}_{1}, \mathfrak{x}_{2}\right\}<\delta$ implies that $\rho\left\{l\left(\mathfrak{x}_{1}\right), l\left(\mathfrak{x}_{2}\right)\right\}<\epsilon(y)$.

Let $\left\{\mathfrak{E}_{k}\right\}$ be a cyclic chain approximation to $\mathfrak{X}$, and select $a$ so large that $n \geqq a$ implies that each component of $\mathfrak{X}-\mathfrak{A}_{n}$ has a diameter less than $\delta / 2$, where $\mathfrak{A}_{n}=\cup \mathfrak{G}_{k}, k=1, \cdots, n$. Consider $\mathfrak{A}_{a}$ and let $\Re_{k}$ be an arc whose end points are "end points" of $\mathfrak{E}_{k}, k=1, \cdots, a$. Let $\mathbb{E} \in \mathcal{E}$ if and only if $\mathbb{E} \in \mathcal{E}\left(\mathfrak{H}_{a}\right), d(\mathbb{E})<\delta / 2$ and $\mathbb{E}_{\mathfrak{C}} \cap \mathfrak{C}_{k} \neq \varnothing$ for exactly one integer $k=1, \cdots, a$. Define $\mathfrak{Q}=\left[\mathfrak{H}_{a}-\cup \mathfrak{E}, \mathfrak{E} \in \mathcal{E}\right] \cup\left[\cup \Omega_{k}\right.$, $k=1, \cdots, a]$.

It is easy to see that $\mathfrak{Q} \in \mathscr{Q}(\mathfrak{X})$, in fact $\mathfrak{A}_{a}$ is the smallest $A$-set containing $\mathfrak{Q}$, and if $\mathcal{E}(\mathfrak{Q}) \boxplus \mathbb{E} \in \mathcal{E}\left(\mathfrak{H}_{a}\right)$, then $\mathbb{E} \cap \mathfrak{Q}$ is an arc.

Now suppose that $\mathfrak{Q} \supset \mathfrak{R} \in \mathcal{Q}(\mathfrak{X})$. If $\mathfrak{A}$ is the smallest $A$-set containing $\mathfrak{R}$, then $\mathfrak{A} \supset \mathfrak{A}_{a}$ and hence each component of $\mathfrak{X}-\mathfrak{A}$ is in some component of $\mathfrak{X}-\mathfrak{A}_{a}$. Moreover, if $\mathcal{E}(\mathfrak{R}) \boxplus \mathbb{E} \in \mathcal{E}(\mathfrak{R})$, then $\mathbb{E} \in \mathcal{E}$ or $\mathbb{E}$ is in some component of $\mathfrak{X}-\mathfrak{A}_{a}$. In either event $d(\mathbb{E})<\delta / 2$. Hence by (3), $\rho\left\{x, r_{\Re}(x)\right\}<\delta$.

Since $f_{\Re}=l_{\Re \Re m}$, the selection of $\delta$ shows that $\rho\left\{f(x), f_{\Re}(x)\right\}$ $<\epsilon(y), x \in X$. Consequently $f^{*}(y)=f_{\Re}^{*}(y)$; that is, $\gamma(f)=\gamma\left(f_{\Re}\right)$.

\section{BIBLIOGRAPHY}

1. Karol Borsuk, Einige Sätze über Streckenbilder, Fund. Math. vol. 18 (1932) pp. 198-213.

2. - Sur l'addition homologique des types de transformations continues en surfaces spheriques, Ann. of Math. vol. 38 (1937) pp. 733-738.

3. R. G. Helsel, A theorem on surface area, Trans. Amer. Math. Soc. vol. 61 (1947) pp. 443-453.

4. W. Hurewicz and H. Wallman, Dimension theory, Princeton, 1941.

5. T. Rad6, On continuous mappings of Peano spaces, Trans. Amer. Math. Soc. vol. 58 (1945) pp. 420-454.

6. G. T. Whyburn, Analytic topology, Amer. Math. Soc. Colloquium Publications, vol. 28, New York, 1941.

INDIANA UNIVERSITY 